\title{
DELIRIUM IN HOSPITALIZED ELDERLY PATIENTS AND POST-DISCHARGE MORTALITY
}

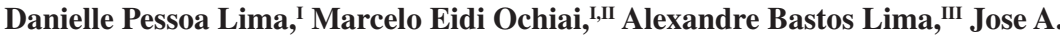 \\ E. Curiati, ${ }^{\text {IV }}$ Jose M. Farfel, ${ }^{\text {IV }}$ Wilson Jacob Filho ${ }^{\text {IV }}$
}

doi: $10.1590 / \mathrm{S} 1807-59322010000300003$

Lima DP, Ochiai ME, Lima AB, Curiati JAE, Farfel JM, Filho WJ. Delirium in hospitalized elderly patients and post-discharge mortality. Clinics. 2010;65(3):251-5.

OBJECTIVE: To determine the impact of delirium on post-discharge mortality in hospitalized older patients.

INTRODUCTION: Delirium is frequent in hospitalized older patients and correlates with high hospital mortality. There are only a few studies about its impact on post-discharge mortality.

METHODS: This is a prospective study of patients over 60 years old who were hospitalized in the Geriatric Unit at Hospital das Clínicas of São Paulo between May 2006 and March 2007. Upon admission, demographics, comorbidities, number of drugs taken, and serum albumin concentration were evaluated for each patient. Delirium was diagnosed according to the DSM-IV criteria. Patients were divided into group A (with delirium) and group B (without delirium). One year after discharge, the patients or their caregivers were contacted to assess days of survival.

RESULTS: The sample included 199 patients, 66 (33\%) of whom developed delirium (Group A). After one year, 33 (50\%) group A patients had died, and $45(33.8 \%)$ group B patients had died $(p=0.03)$. There was a significant statistical difference in average age $(p=0.001)$ and immobility $(p<0.001)$ between groups $A$ and $B$. There were no statistically significant differences between groups $A$ and $B$ in number of drugs taken greater than four $(p=0.62)$, sex $(p=0.54)$ and number of diagnoses greater than four $(p=$ 0.21 ). According to a multivariate analysis, delirium was not an independent predictor of post-discharge mortality. The predictors of post-discharge mortality were age $\geq 80$ years $(\mathrm{p}=0.029)$, albumin concentration $<3.5 \mathrm{~g} / \mathrm{dl}(\mathrm{p}=0.001)$ and immobility $(\mathrm{p}=0.007)$. CONCLUSION: Delirium is associated with higher post-discharge mortality as a dependent predictor.

KEYWORDS: Delirium; Post-discharge mortality; Elderly; Post discharge; Survival.

\section{INTRODUCTION}

Delirium is an acute psychiatric syndrome characterized by a transitory, floating, and important disorganization of cognitive function caused by changes in brain metabolism. It is a frequent problem in hospitalized older patients, with a prevalence of $14-56 \%$, depending on the study, and with a

\footnotetext{
${ }^{\text {I}}$ Hospital Auxiliar de Cotoxó - São Paulo/SP, Brazil.

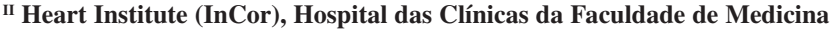
da Universidade de São Paulo - São Paulo/SP, Brazil.

III Instituto de Psiquiatria, Hospital das Clínicas da Faculdade de Medicina da Universidade de São Paulo, São Paulo/SP, Brazil.

Iv Hospital das Clínicas da Faculdade de Medicina da Universidade de São Paulo, São Paulo/SP, Brazil.

Email: dra.daniellelima@gmail.com

Tel.: 55113341.6886

Received for publication October 22, 2009

Accepted for publication on October 26, 2009
}

hospital mortality rate of $25-33 \%{ }^{1}$

In Brazil, hospitalization of patients 60 years old and over is becoming increasingly frequent. From January 2002 to December 2007, $19.4 \%$ of total admissions in the public health system were older than 60 years. Delirium increases morbidity, functional impairment, length of hospital stay, number of hospitalizations, caregiver stress, and financial cost. ${ }^{2}$ In addition, delirium is associated with worse prognosis during hospitalization and after discharge. ${ }^{3-5}$

Despite the fact that delirium has major effects before, during and after discharge, there are few Brazilian studies on delirium. The adverse outcomes associated with prolonged hospital stays and the increased risk of death in patients with delirium are well established. ${ }^{4,5}$ Recently, a Brazilian study found that delirium is a mortality predictor among older patients. ${ }^{6}$

The objective of this study is to evaluate the association 
between delirium and post-discharge mortality in hospitalized older patients.

\section{MATERIALS AND METHODS}

\section{Experimental Design}

This is a prospective cohort study of patients over 60 years old admitted to the Geriatric ward of Hospital das Clínicas - University of São Paulo Medical School between May 2006 and March 2007. Ethical approval for this study was granted by the local research ethics committee (Cappesq - Comissão de Ética para Análise de Projetos).

Pre-defined questionnaires designed by a multidisciplinary team were used to collect participants' data. Gender, age, reason for hospitalization (clinical treatment, diagnostic investigation or palliative care), number of diagnoses, number of drugs in use at the time of admission, heart failure history, neoplastic disease history, infectious disease diagnosis, urinary incontinence, and immobility (defined as the inability to change positions in bed without help) ${ }^{7}$ were evaluated at admission and discharge.

Delirium was diagnosed by DSM-IV criteria: disturbance of consciousness associated with reduced ability to maintain attention and changes in cognition that develop over a short period of time, usually hours to days, with a tendency to fluctuate during the day. This is evidence that the disruption is caused by direct physiological consequences of a general medical condition. ${ }^{8}$ During hospitalization, patients were evaluated daily by the multidisciplinary health team, and any signs of delirium were reported.

Patients were excluded from the study if the length of their hospital stay was less than 48 hours or if it was not possible to obtain information about their post-discharge survival.

The patients were divided into group A (with delirium) and group B (without delirium). The patients or their caregivers were contacted by telephone about twelve months after discharge to determine the days of survival after discharge.

The primary endpoint of this study was mortality from any and all causes. The secondary endpoint was length of hospital stay.

Groups A and B were compared regarding days of survival after hospital discharge. Other variables, such as the number of comorbidities, age, albumin concentration in serum, immobility ${ }^{7}$ and the number of drugs being taken, were also considered.

\section{Statistical analysis}

The continuous variables were expressed as means and standard deviations and analyzed by Student's t tests. The categorical variables were expressed in numbers and proportions and analyzed by Fisher's exact test or the Chisquare test. ${ }^{9}$ A p-value $<0.05$ (two-tailed) was considered significant.

The variables for which the p-value was less than 0.10 were included in multivariate analysis by Cox regression ${ }^{10}$ to calculate the relative risk and the $95 \%$ confidence interval. In the multivariate analysis, the time between discharge and death was used as the outcome. Cox regression was performed using analytical software. The survival curves were constructed using the Kaplan-Meier ${ }^{11}$ method and compared with the log-rank ${ }^{12}$ test. These analyses were performed using SPSS statistical software (Chicago, IL, USA).

\section{RESULTS}

A total of 299 patients were hospitalized in the Geriatric unit of São Paulo University Hospital between May 2006 and March 2007. Of these patients, 100 were excluded (50 died during their hospital stay, 9 were hospitalized for less than or equal to 48 hours, 7 left no contact information, and 34 had hospital records that were not reliable enough to determine the occurrence of delirium during their hospital stay).

The 199 patients took part in the study, a group that had the following characteristics: mean age of 77.9 years, 106 female patients (53\%), and an average period of hospitalization of $17 \pm 14$ days. Of these patients, $115(58 \%)$ were hospitalized for clinical compensation, 76 patients (38.2\%) for diagnostic investigation and 1 patient $(0.8 \%)$ for surgery. One hundred and twenty-five patients $(62.8 \%)$ had five or more diagnoses on admission. Immobility was diagnosed in 62 patients $(31.2 \%)$ on admission. The patients were followed for $449 \pm 22$ days after discharge.

\section{Delirium}

In our study, 66 patients $(33.2 \%)$ in the hospital had delirium (group A), and 133 (66.8\%) did not (group B). Death occurred in 33 patients $(50 \%)$ of group A and 45 $(33.8 \%)$ of group B $(p=0.03)$, as shown in Figure 1 .

Groups A and B were compared regarding gender, age ( 80 years old or over), number of diagnoses (five or more), number of drugs (five or more) and immobility on admission. There was a statistically significant difference in the number of octogenarians (59\% in group A versus $33 \%$ in group $\mathrm{B}, \mathrm{p}=0.001$ ) and in the presence of immobility $(51.5 \%$ in group A versus $21 \%$ in group $\mathrm{B}, \mathrm{p}<0.001)$, as shown in Table 1. 


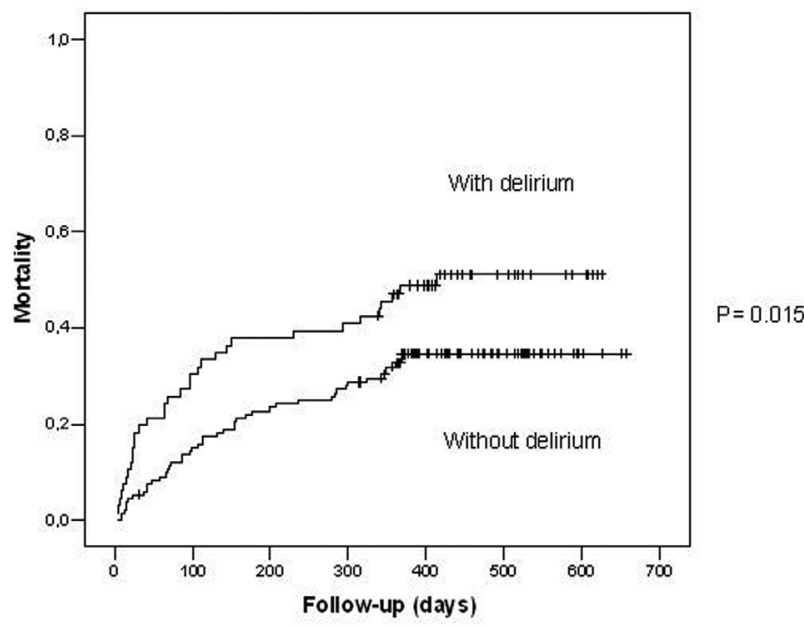

Figure 1 - Mortality curves according to delirium occurrence

Table 1 - Baseline characteristics according to the occurrence of delirium

\begin{tabular}{lccc}
\hline & DELIRIUM & NO DELIRIUM & $\mathrm{P}$ \\
\hline Female gender & $33(50 \%)$ & $73(54.9 \%)$ & 0.54 \\
Age $\geq 80$ & $39(59 \%)$ & $44(33 \%)$ & 0.001 \\
Number of diagnoses $\geq 5$ & $46(69 \%)$ & $79(59.4 \%)$ & 0.21 \\
Number of drugs $\geq 5$ & $32(48.5 \%)$ & $69(51.9 \%)$ & 0.62 \\
Immobility & $34(51.5 \%)$ & $28(21 \%)$ & $<0.001$ \\
\hline
\end{tabular}

In group A, 44 patients $(66.6 \%)$ were admitted with delirium (prevalent delirium), and 22 patients (33.4\%) developed delirium during hospitalization (incident delirium).

\section{Hospitalization}

There were two categories of reasons for hospitalization in this study: urgent causes (clinical compensation) and elective causes (diagnostic evaluation or procedure). We compared the occurrence of delirium across causes of hospitalization (urgent or elective) but found no statistical correlation. In our sample, 122 patients were hospitalized for clinical compensation and 77 for elective reasons. The average number of days of hospitalization were $22.6 \pm 18$ days for group A and $13.8 \pm 11$ days for group B $(p=0.001)$. The mean lengths of hospitalization were $22.6 \pm 18$ days ( $\mathrm{p}$ $=0.001$ ) for patients with prevalent delirium and $25.2 \pm 19$ days $(\mathrm{p}=0.01)$ for patients with incident delirium.

\section{Delirium and mortality}

Multivariate analysis was performed with the variables of age ( $<80$ vs. $\geq 80$ years old), delirium, immobility on discharge, five or more diagnoses on discharge, albumin concentration less than $3.5 \mathrm{~g} / \mathrm{dl}$ on admission, and five or more drugs taken on discharge. An albumin concentration of less than $3.5(p=0.001)$, age $\geq 80$ years $(p=0.03)$ and immobility $(0.007)$ were independent predictors of postdischarge mortality, as shown in Figure 2. Delirium was associated with higher post-discharge mortality, but it was not an independent predictor in this study.

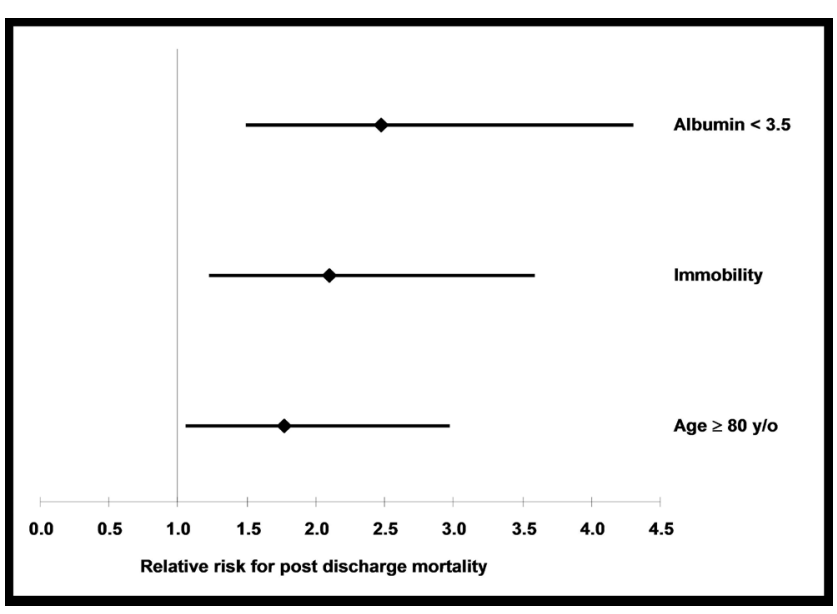

Figure 2 - Relative risk for post-discharge mortality

\section{DISCUSSION}

The incidence of delirium was $33.2 \%$, which is consistent with other studies ${ }^{1,2,4,13}$, but the impact of delirium on post-discharge survival is still a controversial subject. The risk of mortality for delirious patients after one year of hospitalization has been evaluated by other studies ${ }^{13-16}$, namely McCusker et al., who concluded that delirium is an independent predictor of mortality after discharge. ${ }^{16}$ Moreover, the same study found a relative risk of 2.11 (95\% CI, 1.18 to 3.77 ) in a sample of 361 patients evaluated one year after hospital discharge. Leslie et al. found that the risk of mortality in patients who developed delirium in the hospital increased by $62 \%$ twelve months after discharge in a sample of 919 patients who were at least 70 years old and were kept under observation from March 1995 to March 1998. ${ }^{17}$ However, Adamis et al. did not find delirium to be an independent predictor of mortality six months after hospital discharge, ${ }^{18}$ nor did Inouye et al. in a study that examined patients three months after discharge ${ }^{19}$. Several other studies have also found that delirium is not an independent predictor of mortality after discharge. ${ }^{18,23,} 24$ In another study, McAvay et al. showed that delirium upon hospital discharge is an independent predictor of both death and institutionalization when compared with patients who experienced delirium during their hospital stay but recovered before discharge and with those who were not delirious at any time. ${ }^{13}$ 
In our study, delirium was not an independent predictor of post-discharge mortality as assessed by multivariate analysis. The relationship between delirium and postdischarge mortality is still controversial, probably because several different clinical conditions may present themselves as delirium. ${ }^{18,19}$ Another factor contributing to these conflicting results is the heterogeneity of patients admitted to the geriatric care unit. In this study, for example, the ward was used both for clinical compensation and for diagnostic investigation. The reason for hospitalization, however, was not associated with the presence of delirium in the bivariate analysis. This heterogeneity could cause differences in the distributions of multiple factors that are relevant both to delirium and to mortality. ${ }^{18}$

Nevertheless, the occurrence of delirium was significantly more common among patients $\geq 80$ years old $^{20,21}$ and among those diagnosed with immobility. Additionally, delirium was associated with an increased length of hospital stay, which has already been shown in previous studies. . $16,17,19$ The functional decline of patients with delirium, the need for etiological research and the possibility of other underlying medical complications probably contribute to this correlation.

The number of days of survival after discharge was lower for the group with delirium as compared to the group without delirium (294 days vs. 346 days), as shown in other studies. ${ }^{5,16,17,22}$ The independent predictors of late mortality in this study were age $\geq 80$ years old,${ }^{18}$ albumin less than 3.5 at admission ${ }^{25,26}$ and immobility. These predictors have already been cited in other studies. Adamis et al. ${ }^{18}$ found that albumin on admission was an independent predictor of mortality six months after discharge, but in that study, delirium was not found to be a significant predictor. This study also found advanced age was a significant predictor in the bivariate analysis.

\section{Limitations}

Delirium was not actively investigated during hospitalization in a systematic way. It can be underdiagnosed because of its fluctuating course and the occurrence of hypoactive forms. ${ }^{27,28}$ The systematic application of the CAM (Confusion Assessment Method) has been suggested as an important standard in the diagnosis of delirium during hospitalization..$^{29}$

\section{CONCLUSION}

Delirium is associated with higher post-discharge mortality but not as an independent predictor.

\section{REFERENCES}

1. Inouye SK, Schlesinger MJ, Lydon TJ. Delirium: a symptom of how hospital care is failing older persons and a window to improve quality of hospital care. Am J Med. 1999; 106:565-73.

2. Flacker JM, Marcantonio ER. Delirium en the Eldery: Optimal Management. Drugs \& Aging. 1998;2:119-30.

3. Rabins PV, Folstein MF. Delirium and dementia: diagnostic criteria and fatality rates. Br J Psychiatry. 1982;140:149-53.

4. Pompei P, Foreman M, Rudberg MA, Inouye SK, Braund V, Cassel CK. Delirium in hospitalized older persons: outcomes and predictors. J Am Geriatr Soc. 1994;42:809-15.

5. Lin SM, Liu CY, Wang CH, Lin HC, Huang CD, Huang PY, et al. The impact of delirium on the survival of mechanically ventilated patients. Crit Care Med. 2004;32:2254-9.

6 Silva TJ, Jerussalmy CS, Farfel JM, Curiati JA, Jacob-Filho W. Predictors of in-hospital mortality among older patients. Clinics. 2009;64:613-18.

7. Studenski S. Mobility. In: Hazzard WR, Blass JP, editors. Principles of Geriatric Medicine \& Gerontology. 5th ed. McGraw-Hill Companies.2003; p. 947-60.

8. American Psychiatric Association- Diagnostic and Statistical Manual of Mental Disorders (DSM-IV), fourth edition. 1994

9. Kass, GV. An Exploratory Technique for Investigating large Quantities for Categorical Data, Applied Statistical, 1980.
10. Cox DR, Oakes D. Analysis of survival data. Chapman and Hall $1^{\mathrm{ST}}$. Ed., London, 1984.

11. Kaplan EL, Meier P. Nonparametric estimation from incomplete observations. J Am Stat Assoc. 1958;53:457-81.

12. Lakatos E. Sample Sizes Based on the Log-Rank Statistic in Complex Clinical Trials. Biometrics. 1988:44:229-41.

13. McAvay G, Bogardus STJ, Zhang Y, Leslie D, Leo-Summers LS, Inouye SK. Older Adults Discharged from the Hospital with Delirium: 1-Year Outcomes. J A Geriatr Soc. 2006;54:1245-50.

14. Francis J, Kapoor WN. Prognosis after hospital discharge of older medical patients with delirium. J Am Geriatr Soc. 1992;40:601-6.

15. Edelstein DM, Aharonoff GB, Karp A, Capla EL, Zuckerman JD, Koval KJ. Effect of postoperative delirium on outcome after hip fracture. Clin Orthop Relat Res. 2004;422: 195-200.

16. McCusker J, Cole M, Abrahamowicz M, Primeau F, Belzile E. Delirium predicts 12-month mortality. Arch Intern Med. 2002;162:457-63.

17. Leslie DL, Zhang Y, Holford TR, Bogardus ST, Leo-Summers LS, Inouye SK. Premature Death Associated with Delirium at 1-year Followup. Arch Intern Med. 2005;165:1657-62.

18. Adamis D, Darwiche F, Gregson N, Macdonald A, Martin F. Associations of delirium with in-hospital and in 6-months mortality in elderly medical inpatients. Age \& Ageing 2007;36:644-9. 
19. Inouye S, Rushing J, Foreman M, Palmer R, Pompei P. Does delirium contribute to poor hospital outcomes? A three-site epidemiologic study. J Gen Intern Med. 1998;13:234-42.

20. Iseli RK, Brand C, Telford M, LoGiudice D. Delirium in eldery general medical inpatients: a prospective study. Internal Medicine Journal. 2007; 37:806-11.

21. Goldenberg G, Kiselev P, Bharathan T, Baccash E, Gill L; Madhav V, et al. Predicting post-operative delirium in elderly patients undergoing surgery for hip fracture. Psychogeriatrics. 2006;6:43-8.

22. Curyto KJ, Johnson J, TenHave T, Mossey J, Knott K, Katz IR. Survival of hospitalized elderly patients with delirium: a prospective study. Am J Geriatr Psychiatry. 2001;9:141-7.

23. Francis J, Martin D, Kapoor WN. A prospective study of delirium in hospitalized elderly. Jama. 1990;263:1097-101.

24. Levkoff SE, Evans DA, Liptzin B, Cleary PD, Lipsitz LA, Wetle TT, et al, Delirium. The occurrence and persistence of symptoms among hospitalized elderlypatients. Arch Intern Med. 1992;152:334-40.
25. Iwata M, Kuzuya M, Kitagawa Y, Iguchi A. Prognostic value of serum albumin combined with serum C-reactive protein levels in older hospitalized patients: continuing importance of serum albumin. Aging Clin Exp Res. 2006;18:307-11.

26. Reuben DB, Ferrucci L, Wallace R, Tracy RP, Corti MC, Heimovitz H, et al. The prognostic value of serum albumin in healthy older persons with low and high serum interleukin-6 (IL-6) levels. J Am Geriatr Soc. 2000;48:1404-7.

27. Cole M, McCusker J, Dendukuri N, Han L. The Prognostic Significance of Subsyndromal Delirium in Elderly Medical Inpatients. J A Geriatr Soc. 2003;51:754-60

28. Schuurmans MJ, Duursma SA, Shortridge-Baggett LM. Early recognition of delirium: review of the literature. Journal of Clinical Nursing. 2001;10:721-9.

29. Inouye SK, Van Dyck CH, Alessi CA, Balkin S, Siegal AP, Horwitz RI. Clarifying confusion: The Confusion Assessment Method. A new method for detection of delirium. Ann Intern Med. 1990;113:941-8. 
